Images du travail, travail des images

6-7| 2019

Femmes au travail\&nbsp: quelles archives visuelles ?

\title{
Rêves de chaîne : retour sur la réalisation d'un documentaire sociologique
}

Assembly line dreams : reflections on making a sociological documentary

Jean-Pierre Durand et Joyce Sebag

\section{OpenEdition}

\section{Journals}

Édition électronique

URL : https://journals.openedition.org/itti/630

DOI : $10.4000 /$ itti.630

Éditeur

Université de Poitiers

\section{Référence électronique}

Jean-Pierre Durand et Joyce Sebag, «Rêves de chaîne : retour sur la réalisation d'un documentaire sociologique », Images du travail, travail des images [En ligne], 6-7 | 2019, mis en ligne le 01 février 2019, consulté le 21 septembre 2021. URL : http://journals.openedition.org/itti/630 ; DOI : https://doi.org/ $10.4000 /$ itti.630

Ce document a été généré automatiquement le 21 septembre 2021.

Images du travail, travail des images 


\section{Rêves de chaîne : retour sur la réalisation d'un documentaire sociologique}

Assembly line dreams : reflections on making a sociological documentary

Jean-Pierre Durand et Joyce Sebag

1 Cet article revient sur le processus de réalisation d'un film, Rêves de chaîne, documentaire sociologique de 26' que nous avons réalisé au début des années 2000 dans l'usine de montage automobile Nummi à Fremont (Californie). Il s'agit d'une jointventure Toyota-General Motors installée en 1984 dans l'ancienne usine GM fermée à la suite de la crise américaine de l'industrie automobile des années 1980-1982. Dire que nous avons réalisé d'un bout à l'autre ce film - en coopération avec Jean-Pierre Lenoir au moment du montage - signifie qu'il ne s'agit pas d'images d'enquête, puisque la longue enquête avait été faite avant le tournage, mais du résultat d'un processus complet de production des images et des sons, du tournage à la post-production, s'appuyant sur une démarche sociologique. Cet article adopte une attitude réflexive dans la mesure où nous présentons de manière détaillée les écueils et les solutions adoptées pour y faire face dans les différentes étapes de cette réalisation: nous interrogeons nos pratiques concrètes de chercheurs qui allient investigation de terrain (ici dans l'atelier) et théories de sociologie du travail (par exemple la critique de la lean production) en recourant à un autre medium que le papier pour nous exprimer. Le lecteur y trouvera donc des préoccupations pratiques sur l'utilisation de la vidéo en milieu industriel et des questions de méthode (comme par exemple comment rendre compte par l'image des faits intangibles) qui rejoignent les interrogations épistémologiques des sociologues quant à l'usage des données de terrain et à leur mise en rapport avec les théories en cours.

2 Dans ce long processus de production de connaissances qui va de la pré-enquête au montage du documentaire, des événements imprévus viennent perturber régulièrement le cheminement planifié du travail de chercheur : mais le surgissement de ces faits inopinés est peut-être encore plus fréquent ici en raison de l'utilisation de 
la caméra qui pousse les personnes filmées à faire parler leur imaginaire. En même temps, les sociologues-réalisateurs rencontrent d'heureuses surprises, en particulier dans les déclarations claires, vécues et fortement argumentées des ouvriers-monteurs ou dans des images/sons découvertes durant le dérushage qui en disent plus que ce qui avait été perçu au tournage. Ainsi, une team leader, qui explique comment elle s'est imposée face aux ouvriers, révèle la nature des rapports de genre dans l'atelier : un machisme "bon enfant", mais bien réel. Nous discutons ainsi les avantages et les atouts de l'image et du son dans le processus filmique : comment les micro-événements, qui surgissent en permanence dans la conduite du tournage jusqu'au montage, sont traités pour ne pas faire abandonner la ligne directrice de la recherche (problématique et hypothèses) et pour produire du sens et des connaissances à chaque fois que cela est possible?

Le support papier utilisé ici permet de revenir sur les choix d'expression de la réalité que nous, sociologues-réalisateurs, avons effectués. Y compris parce qu'ils donnent à voir, en creux, certains des présupposés portés par les chercheurs. Autant d'analyses qui ne peuvent être présentes dans le film - comme elles ne le sont pas, d'ailleurs, dans l'exposé livresque des résultats de toute recherche - mais qui animent ce texte en montrant combien le texte et l'image/son sont complémentaires en organisant la production de connaissances dans un éternel va-et-vient. Après un retour sur l'enquête sociologique dans l'usine californienne, nous analyserons les voies par lesquelles nous avons maîtrisé ou contourné les obstacles à la réalisation du film, lesquelles ont influencé l'écriture du scénario et le tournage. Dans un troisième moment nous reviendrons sur ce qu'est un entretien filmé, puis comment le dérushage est un instant créatif à valoriser comme conditionnant l'efficacité du montage. Nous pourrons alors affiner quelques principes fondateurs de la sociologie filmique tout en rappelant que cet article n'entend pas répondre à toutes les questions qu'il soulève !

\section{L'enquête sociologique : la lean production en Californie}

4 Filmer un objet sociologique exige de bien le connaître afin d'adopter les points de vue (la place de la caméra) et les cadrages qui montrent le mieux ce que souhaite dire le sociologue-réalisateur. En même temps, l'enquête sociologique préalable au tournage ne signifie pas que le tournage et plus généralement le film sociologique ne sont qu'un mode d'expression particulier d'un résultat de recherche. Bien au contraire, la production de connaissances a lieu tout au long du processus de réalisation du documentaire sociologique, à condition de bien maîtriser son terrain et les théories sociologiques afférentes. D'où ce court retour historique sur les conditions d'entrée dans cette usine particulière qui a été notre terrain.

5 À la fin des années 1980 , l'un d'entre nous dirigeait, pour le compte du GERPISA ${ }^{1}$, un programme de recherche sur l'évolution des relations de travail dans l'industrie automobile à la suite de la mise en œuvre des principes de la lean production. Comme on le sait mieux aujourd'hui, ces principes de la "production frugale» visent à réduire considérablement les coûts de production, de l'ingénierie (réduction du nombre de pièces et meilleure manufacturabilité de celles-ci) à leur fabrication et à leur montage (Womack et al, 1992). L'organisation du travail est profondément remaniée avec la généralisation du flux tendu, c'est-à-dire avec la disparition des encours dans la 
production qui permettaient précédemment à chacun de réguler plus ou moins le rythme de son travail (Durand, 2004). De nouveaux outils de gestion du travail au cœur de la production, tels que le travail en groupe, les cercles de qualité, la maintenance totale, le kaizen (amélioration permanente des systèmes productifs) transforment les rapports des ouvriers à la matière, mais aussi leurs relations entre eux ou avec leur hiérarchie. Même les espaces de travail évoluent : si les lieux de production eux-mêmes rétrécissent (la réduction des coûts des bâtiments est devenue un impératif), l'émergence de salle de repos, d'espaces réservés aux pauses modifient quelque peu la perception du travail par les ouvriers.

6 Enfin, l'invention du team leader qui prend la place du chef d'équipe témoigne d'un autre changement dans la responsabilisation des ouvriers face à leurs tâches (ce qui va de pair avec l'autocontrôle de la qualité du produit et la réduction notoire du nombre de contrôleurs qualité), voire d'un certain accroissement de leur autonomie : en effet, la complexification des process et leur fragilisation par la disparition des encours (stockstampons), qui hier palliaient les aléas dans l'atelier, exigent à la fois un travail collectif et l'assurance qu'il sera bien réalisé. Le team leader, devenu moniteur ou animateur en français, n'a pas de responsabilité hiérarchique sur les team members (les ouvriers de base) et doit les convaincre des bonnes pratiques à adopter: il est un membre du groupe comme les autres, assure en partie les mêmes tâches (ici de montage et d'habillage des voitures) et veille à ce que son groupe dispose d'un outillage en bon état; de plus il gère les absences et les remplacements, organise les plans de formation. Il bénéficie d'une maigre prime pour toutes ces fonctions (entre 5 et $10 \%$ du salaire de base des ouvriers) qui l'obligent à arriver avant ses collègues et à partir après eux. Si la plupart des team leaders acceptent ce statut, c'est essentiellement parce qu'ils y perçoivent une reconnaissance par leur hiérarchie qui peut les conduire à d'autres fonctions mieux rémunérées et plus intéressantes tout en les faisant sortir des contraintes et des pressions du flux tendu que subissent les ouvriers de montage. L'importance stratégique du team leader (quelle que soit la dénomination qu'il endosse) réside dans le fait que sa position (il appartient au groupe de travail tout en ayant des liens privilégiés avec la ligne hiérarchique) rétablit la communication entre les échanges informels horizontaux du groupe ouvrier et la verticalité des informations issues de la ligne de commandement. Or cette communication était en général très mauvaise dans la production fordienne, la trop grande prégnance de la fonction hiérarchique contrariant les échanges informels nécessaires à la réalité de la production.

7 Toutes ces transformations font conclure la plupart des experts à une transformation du travail qui le rend à la fois plus intéressant - au moins provisoirement car il est probable que les effets de ces innovations s'essoufflent aujourd'hui - et plus dur en raison des pressions temporelles déjà citées nées du flux tendu. L'un des projets de recherche du GERPISA consistait en la comparaison des transformations du travail dans vingt sept ateliers de montage automobile sur quatre continents, en fonction des traditions nationales et industrielles de chaque groupe automobile concerné. En effet, si les principes de la lean production régnaient sans partage dans la direction des groupes, leur mise en œuvre dépendait quelque peu de la «culture d'entreprise » de ceux-ci, mais surtout des terrains d'application. Les questions de droit du travail, du degré d'influence des syndicats, de la tradition plus ou moins taylorienne des établissements, des modes nationaux de rémunération, des niveaux de qualification, de la moyenne d'âge des ouvriers influaient sur les modalités de mises en œuvre des 
principes généraux de la lean production. C'est ainsi que nous avons pu mettre en évidence cinq « modèles » d'organisation et de relations de travail qui croisaient huit variables principales (Durand, Stewart et Castillo, 1998).

Selon nos lectures et nos échanges dans ce groupe international rassemblant plusieurs dizaines de chercheurs, l'usine NUMMI de Fremont en Californie correspondait à une interprétation locale du lean management qui avait considérablement durci les conditions et les rythmes de travail pour les ouvriers ayant travaillé chez GM quelques années auparavant. Par exemple, pour être embauchés ou ré-embauchés ${ }^{2}$ tous les salariés ont dû signer un contrat qui imposait le respect des principes du « système de production Toyota » (autrement dit la lean production) et excluait le droit de grève. Or, le chapitre du collègue américain Paul Adler, très favorable au "modèle japonais " contre le "modèle uddevallien" mis en place par Volvo dans l'une de ses usines, percevait dans les transformations du travail imposées par la direction de NUMMI de nouvelles voies pour humaniser le travail ouvrier et en particulier le travail d'exécution. Son chapitre avançait le terme de «taylorisme démocratique " pour caractériser l'intérêt au travail qu'il disait avoir rencontré chez les ouvriers (Adler, 1998). Nous avions un doute sur l'interprétation des données recueillies, y compris parce que le travail de terrain ne paraissait guère conséquent par rapport à ce que d'autres auteurs avaient réalisé dans leurs ateliers : ne s'agissait-il pas d'abord de la reprise du discours de la direction qui avait fait de NUMMI une sorte d'exemple que devrait suivre les autres usines pour "se moderniser"? Il y avait un précédent américain - l'usine Saturn, division de GM diffusant sous la marque Saturn un seul véhicule de milieu de gamme - dont nous avions déjà montré l'écart entre les annonces managériales reprises par nombre de commentateurs (Lorino, 1987) et la réalité du travail ouvrier après une visite et de multiples rencontres avec les ouvriers. Ainsi, afin d'homogénéiser les modes de recueil des données - ce que nous avions déjà fait pour certaines usines suédoises et japonaises -, nous sommes partis à la rencontre de NUMMI.

9 Ce premier séjour à l'usine de Fremont a été particulièrement intensif puisque nous l'avions préparé avec la direction et avec des représentants syndicaux. Les entretiens avec la direction ont confirmé nos lectures sur la volonté de rénover les relations professionnelles et l'organisation du travail pour en accroître la productivité immédiate. L'ouverture de la joint-venture comblait toutes les attentes des deux constructeurs : celles de GM qui apprenait en direct, avec des représentants de Toyota, comment mettre en place la lean production et celles de Toyota qui élargissait sa base productive au cœur du marché du continent américain. La visite de l'usine, très orientée "productivité du travail et syndicalisme » nous a permis d'avoir de premiers entretiens avec des managers, des leaders syndicaux, des ouvriers de base et quelques team leaders. La qualité de l'accueil par les dirigeants de l'usine et par les syndicalistes autant que l'intérêt suscité par l'exemplarité de la mise en place de la lean production a fait rapidement germer en nous l'idée d'y réaliser un documentaire sociologique.

De plus, certains moments de la visite et contenus des entretiens avivaient notre souhait de poursuivre l'enquête. Les syndicalistes soulignaient combien ils avaient dû reculer sur l'essentiel (le droit de grève) pour avoir le droit d'exister dans l'usine et comment ils tentaient de résister à l'accroissement des cadences de travail dans les ateliers de montage. Nous avions aussi rencontré une femme militante de la génération pour laquelle la libération des ouvriers ne pouvait passer que par l'action collective (de 
masse) qu'elle avait bien du mal à organiser. Par ailleurs, parmi les team leaders, une jeune femme n'avait cessé de valoriser l'engagement au travail comme source de promotion individuelle. Cette dernière, ne correspondait pas par son allure générale et sa tenue à la vision traditionnelle de l'ouvrière. Son originalité pouvait en faire un personnage important dans la narration d'un film, se différenciant de la militante qui investissait dans l'action collective. Ces deux personnages offraient deux conceptions très différentes de la sortie de l'aliénation du travail ouvrier : la solution individuelle pour échapper aux contraintes de la ligne de montage et la voie revendicative ou collective pour transformer le travail en le rendant plus acceptable pour les ouvriers. C'était un beau projet qui alliait ressort narratif et démonstration sociologique quant à la nature de la lean production.

11 Ainsi, à n-11 mois du tournage - qui n'était pas encore fermement décidé - nous disposions de solides connaissances sur notre objet: une théorie critique de la lean production (les effets du sans stock sur le travail avec le rôle clé des team leaders dans la nouvelle organisation) et une connaissance de l'usine de Fremont dont les éléments matériels ne différaient guère des dizaines d'usines que nous avions visitées à travers le monde. La reprise de contacts avec la direction de l'usine a eu lieu via un cadre français qui s'était installé en Californie et qui travaillait à la Direction de la Communication de NUMMI. L'exposé de la première version de notre demande d'autorisation de tournage rencontra un accueil favorable et nous avons eu une dizaine de mois pour préparer notre séjour dans l'usine et pour négocier nos demandes. Il ne s'agissait pas pour nous de proposer un scénario à proprement parler, mais une suite d'intentions qui reprenaient l'esprit de l'ouvrage collectif du GERPISA sur la connaissance de la réalité du travail ouvrier dans une usine japonisée. Nous souhaitions disposer d'une douzaine de journées de tournage dans l'atelier où se trouvait la team leader dont nous voulions nous assurer la coopération, avec des entretiens libres dans une salle adjacente à l'atelier (trop de bruit pour filmer les entretiens dans l'atelier) avec des team members et des team leaders, des chefs de groupe (premier niveau hiérarchique manageant les team leaders) et des représentants de la direction expliquant la stratégie de l'entreprise en matière de gestion du travail.

Parallèlement à ces échanges avec la direction, nous avions pris contact avec le syndicat, l'United of Automobile Workers Union (UAW). Ici, il faut distinguer la section syndicale avec sa hiérarchie élue d'une part et les négociateurs qui traitent avec la direction d'autre part. Traditionnellement les seconds sont plus durs dans leur opposition aux directions. Si nous étions en contact avec les deux types de représentants, notre souhait de "démonter » le fonctionnement de la lean production qui commençait à se généraliser en France - nous conduisait à privilégier les négociateurs, y compris parce que nous avions été introduits auprès d'eux par la militante activiste que nous voulions voir figurer dans le film. Parce qu'une partie des syndicalistes sont installés dans les locaux de la Direction des ressources humaines - ce qui paraît invraisemblable de ce côté de l'Atlantique - l'un de nos fax contenant une première version du guide d'entretien prévu avec les négociateurs a été vu ou intercepté par les services de la DRH. Immédiatement après l'envoi de ce fax, il nous a été demandé - nous étions à deux mois du tournage - de ne plus avoir de contact avec la fraction la plus radicale des négociateurs, sous peine de ne pas avoir accès à l'usine. C'était notre première déception avec la nécessité de nous plier à l'injonction si nous voulions faire le film; ce qui ne nous empêchait pas de rester en contact avec la femme 
militante, mais l'esprit dans lequel nous abordions à nouveau le terrain s'en trouvait modifié.

Tout en conservant la rigueur du cadre scientifique dont nous disposions, il nous fallait songer à réorienter la narration car nous étions de moins en moins certains que le vaet-vient entre les deux personnages féminins soit possible, eu égard aux récentes réactions de la direction de l'entreprise: notre espoir de pouvoir choisir les personnages principaux s'estompait peu à peu. En regardant tous les possibles, il nous fallait recentrer le film sur le travail ouvrier, sur son évolution et sur le rôle des team leaders pour faire ressortir le double caractère des transformations du travail : à la fois plus intéressant (smarter) avec des défis de productivité lancés aux ouvriers et plus dur (harder) en raison de cadences plus lourdes. Le problème résidait dans la difficulté de rendre par l'image, la pression par le temps imposée aux ouvriers. En même temps, nous avions bien conscience que nous avions perdu le cœur de la narration (le contraste entre les deux femmes) : ne souhaitant pas abandonner le projet, il nous restait un bel objet scientifique, les moyens d'obtenir un riche matériau (les autorisations de la direction, l'acceptation garantie d'ouvriers et d'ouvrières, puis de syndicalistes et de membres de la direction), mais nous n'avions plus réellement de ressort pour faire vivre le film au montage. Tant pis! Juillet, mois du tournage se rapprochait et nous sommes allés à Fremont sans avoir réellement résolu cette question narrative.

\section{Filmer dans l'atelier}

Accueillis par la Direction de la Communication comme c'est la tradition dans toute l'industrie, nous avons dû renégocier le projet car celle-ci ne faisait guère la différence entre une équipe de télévision et une équipe d'universitaires. Il fallait à la fois allonger la durée du temps de tournage que la direction envisageait sur une semaine et s'assurer de notre liberté de choisir les ouvriers à interviewer. Puis nos interlocuteurs nous ont informé que le tournage n'aurait pas lieu dans l'ancienne usine, mais dans le tout nouvel atelier qui produisait le pick up Tacoma : c'était donc la fin de tout espoir de rencontrer à nouveau la jeune team leader et son contraire, l'activiste, sur le même lieu de travail. C'était aussi un risque de nous trouver en face de rythmes de travail inférieurs à ceux que connaissent les ouvriers sur de plus petits véhicules populaires à plus faible image, fabriqués dans le vieil atelier. Mais aucune négociation n'est apparue possible quant au choix de l'atelier Tacoma.

Dès la visite de l'atelier réalisée - un atelier moderne, très propre, avec un outillage performant $^{3}-$, nos interlocuteurs nous ont demandé de définir notre champ d'action sur la ligne qu'ils avaient choisie (plus de soixante mètres de long et environ une quarantaine d'ouvriers, team leaders compris): combien d'ouvriers/ouvrières souhaitez vous interroger? Préférez-vous qu'ils fassent partie d'une ou de plusieurs équipes? Que souhaitez-vous filmer maintenant que vous connaissez l'atelier? Il nous est apparu immédiatement que les réponses fournies allaient engager les conditions $\mathrm{du}$ tournage car nos accompagnateurs édictaient au responsable de l'atelier les règles et les limites de notre intervention. Notre connaissance des situations de travail sur de telles lignes de montage nous a conduits à ne pas nous disperser sur toute la ligne, mais à choisir une seule équipe avec laquelle nous pourrions tisser des liens plus 
prometteurs. Nous avons aussi obtenu de pouvoir interroger des team leaders animant d'autres équipes, puis le chef de groupe en situation de travail et en entretiens.

L'introduction sur le terrain n'a pas été facile car nous avons été immédiatement livrés à nous-mêmes, devant expliquer à chacun le sens de notre présence et le projet en cours, tout en demandant l'autorisation orale à chaque ouvrier de l'inclure dans le film quand il travaille et si possible de nous entretenir avec lui dans une salle de réunion adjacente qui nous avait été dédiée. Voici donc un paradoxe de plus : alors que nous craignions d'être téléguidés vers des ouvriers/ouvrières peu critiques envers l'organisation du travail, nous étions abandonnés à un groupe qui ne connaissait rien de nous et dont nous devions conquérir très rapidement la confiance. Nous avons certainement bénéficié ici de notre statut d'étranger au regard de l'accueil chaleureux et intéressé qui nous a été réservé y compris dans les réponses à des questions plutôt intimes sur leur perception du travail. Seule, une femme (sur une douzaine de team members) a refusé de participer au tournage au nom du respect de sa vie privée. Tous les feux étant au vert, nous avons choisi de tourner le lendemain matin de notre arrivée, sans faire d'entretiens, en privilégiant les gestes des ouvriers et en jouant sur les couleurs vives des pick up frappés par la violence des éclairages artificiels.

\subsection{Faire sens par les images}

Rapidement, nous avons été confrontés au problème de tout cinéaste filmant des cadences de travail élevées : comment en rendre compte? Car les gestes manuels sont « coulés » (Durand et Hatzfeld, 2001) au sens où la dextérité des ouvriers et la volonté de «s'économiser» pour tenir sur le long terme (la journée, le mois, l'année) les conduisent à accomplir un minimum de mouvements et de déplacements pour un maximum d'efficacité. Autrement dit, c'est seulement en expérimentant ce travail posté que l'on se rend compte de sa difficulté. Par le texte, le sociologue s'attache à décrire le rythme rapide du travail que le lecteur peut alors imaginer à partir de ses acquis sur l'organisation fordienne. Le réel filmé propose « objectivement » un rythme qui apparait lent au spectateur, en raison de la dextérité ouvrière. Comment le film peut-il alors rendre compte, sans truquage et sans artifice (telles les accélérations propres aux premiers films burlesques qui «font autorité » dans la dénonciation du travail à la chaîne), du fort rythme de travail en partie compensé par « l'intelligence de la main »? La réponse est pour nous difficile tant la maîtrise du poste par celui qui l'occupe est la condition de survie sur une ligne de montage ${ }^{4}$. Nous nous sommes quand même attachés à montrer l'enchaînement rapide des gestes dans quelques plans, mais l'exercice n'est probant, pourrait-on dire, que pour les spécialistes du travail ouvrier qui perçoivent immédiatement l'intensité du travail. Le lecteur peut regarder les plans ci-dessous qui montrent en temps réel sur quelques dizaines de secondes combien de gestes sont enchaînés pour engager puis visser des boulons avec des outils au maniement difficile, tant l'extrême longueur de l'embout exige une grande habileté. Par l'attention que nous lui demandons d'accorder à de tels plans, le spectateur peut être convaincu de la justesse du plan montrant l'intensité du geste ; mais en est-il de même s'il regarde le film dans sa totalité sans avoir été sensibilisé à ce thème des conditions de travail ? Plus encore, la question de la perception de la rapidité des gestes par le spectateur se trouve perturbée par les impératifs de montage du film : si les plans sont trop didactiques, trop ethnographiques ou tout simplement trop « ergonomiques » parce qu'ils exigent une certaine longueur afin de bien montrer l'intensité du travail, 
ils entrent en contradiction avec le rythme du film et de sa narration : ils sont soit éliminés, soit raccourcis, perdant ainsi beaucoup de leur dimension démonstrative...

Ce média ne peut être affiché ici. Veuillez vous reporter à l'édition en ligne http:// journals.openedition.org/itti/630

Le second thème à mettre en images était celui de la répétitivité des opérations et des gestes sur un temps de cycle d'environ une minute. Le problème est qu'une minute en temps réel n'est (presque) jamais rendue en une minute complète au cinéma. L'une des caractéristiques du cinéma est de concentrer les durées : une journée ou une heure d'action sont réduites à quelques minutes. Ce qui est devenu un code cinématographique empêche de porter à l'écran la durée réelle d'une opération : une minute apparaitrait infiniment lente au spectateur qui percevrait alors un travail effectué tranquillement par des ouvriers sous-utilisés. Enfin, la répétition de 2 ou 3 minutes des mêmes tâches répétitives en temps réel briserait tout autant le rythme du film et en transformerait la nature. Ainsi, pour montrer la répétitivité - déjà présente dans le vissage où chaque monteur fixe plusieurs vis dans la minute de cycle - nous avons glissé de celle-ci vers la sérialité, à la recherche d'attributs qui montrent la longue série et les grands nombres. Nous avons donc choisi de filmer des caisses de boulons et des caisses d'écrous, avec la main d'une ouvrière qui en remplissait ses poches avant de rejoindre la ligne de montage. Dans l'atelier, nous tournions avec deux caméras, sans pied ou stabilisateurs, ni micros supplémentaires afin d'être plus mobiles : il s'agissait de trouver à chaque fois le point de vue, donc la position corporelle adéquate pour capter au mieux les gestes, les ambiances d'atelier et tous les détails qui font sens : personnalisation d'espaces avec des photos de famille, suivi papier ou informatique de la production et des encours... Chaque opérateur choisissait la longueur de ses plans en fonction de l'objet filmé et du sens recherché : précision du geste, répétitivité, dextérité du monteur, occupations pendant le temps libéré ou pendant les (rares) pannes, etc. Nous échangions systématiquement, pendant nos pauses, des informations sur ce que nous avions filmé afin de stimuler notre créativité, sachant que le temps de tournage était limité.

Un troisième thème encore plus difficile à mettre en images était celui de la dextérité des ouvriers-monteurs. Nous avons retenu le placement du pare brise par deux ouvriers (un de chaque côté du véhicule) guidant un système d'aide à la manutention. Ici, il s'agit, pour les deux monteurs, de coordonner leurs gestes afin de le placer du premier coup au bon endroit à un millimètre près : le pare brise étant collé, toute fausse manœuvre ralentit considérablement le rythme puisqu'il faut tout réajuster en le retirant pour le replacer. L'image montre une hésitation d'une fraction de seconde du duo de monteurs, une sorte d'arrêt en l'air, avant le plaquage sur la voiture (et sur la colle du vitrage). Cet arrêt extrêmement court du mouvement traduit à la fois la difficulté de l'opération, la nécessaire dextérité des ouvriers et leur entente ou leur coopération précise pour que l'ajustement ait lieu. Une fois de plus, le risque est que seuls les habitués et les professionnels de la fabrication automobile perçoivent, dans ce mouvement à la fois coulé et arrêté tout le savoir-faire, la précision, la préparation physique et mentale, en fin de compte l'engagement nécessaire à la tenue de ce poste.

21 Dans le même sens, un plan montre un ouvrier en train de courir, en arrière-plan : pour le profane, cette image n'a guère de sens. Pourtant, cette pression par le temps est permanente et la course n'est pas un acte rarissime : s'il manque des pièces dans un bac 
ou si une information est urgente à donner à l'autre bout de la ligne, l'un des ouvriers est désigné par un team leader pour répondre à l'urgence. L'organisation est tellement tendue que la seule variable d'ajustement est l'accélération de l'activité humaine. Pourquoi ne pas multiplier alors les plans montrant des ouvriers courant dans l'atelier y compris pour donner un rythme au film lui-même par un montage adroit ? Parce qu'il est quasiment impossible de montrer pourquoi ils courent et où ils vont, tant ils démarrent et reviennent rapidement : ce qui enlèverait toute signification aux images et aux mouvements browniens et risquerait d'égarer les spectateurs.

\subsection{De la qualité des images et des sons}

L'usine est extrêmement bruyante en raison de l'outillage lui-même auquel s'ajoute le bruit des convoyeurs et des descenseurs-ascenseurs des caisses automobiles. Les klaxons actionnés par les ouvriers en difficulté qui demandent de l'aide pour ne pas arrêter la chaîne en font un milieu assourdissant qui empêche de procéder aux entretiens dans l'atelier lui-même. Une séquence d'atelier montre le chef de groupe insistant sur l'amplitude de ses responsabilités d'un bout à l'autre de la ligne : fallait-il conserver cette séquence dont le bruit couvre sa voix qu'il a pourtant très puissante et très grave ? N'y avait-il pas le risque de voir les spectateurs juger de la mauvaise qualité du son (et du film) alors qu'il s'agissait de mettre en relief le niveau sonore de l'atelier? Ainsi, la bande-son a toute son importance pour exprimer les conditions de travail et les difficultés de communication alors que nous sommes dans un processus fragilisé par l'absence d'encours, reposant sur un travail collectif. En même temps, la lecture de cette bande-son est elle-même difficile pour le spectateur : sait-il que ces klaxons qui se suivent en permanence sont des appels d'ouvriers à leurs collègues pour résoudre immédiatement un problème qui risque d'arrêter la ligne de montage ? C'est le bruit de l'urgence, mais comme il est devenu ordinaire, le spectateur non-averti ne l'entend pas...

Ce média ne peut être affiché ici. Veuillez vous reporter à l'édition en ligne http:// journals.openedition.org/itti/630

En résumé, l'objectif est de concevoir le sens des images (et des sons) au tournage. Ce qui signifie faire converger au moins quatre préoccupations pour que «monte» la signification souhaitée dans les images :

- La composition des images (valeur des plans, cadrage, couleurs; ici il faut aussi prendre en compte la préoccupation esthétique de l'image).

- Les mouvements de caméra (et quelques fois de zoom, si nécessaire)

- Le choix du point de vue (position de la caméra par rapport à l'objet filmé)

- Le choix des lumières (quand c'est possible).

Autant d'éléments qui demandent quelques précisions. Car le son, le cadrage, les mouvements de caméra, la lumière font aussi partie d'un souci esthétique de l'écriture cinématographique. Lequel traverse chacun des projets et accompagne la construction du dispositif. En ce qui concerne le son, si nous nous en tenons à notre exemple, nous avons choisi de débattre avec les ouvriers en dehors de leur atelier beaucoup trop bruyant. L'esthétique ici correspondait à un choix que nous pourrions qualifier de « moral » puisqu'il n'était pas question de brader une parole à la fois rare et précieuse qui se fait peu entendre en tant que vécu et qui apportait plus de justesse à nos travaux. 

donne des images souvent verdâtres. Devions-nous changer la température de couleur à l'étalonnage ? Nous l'avions envisagé et nous nous étions posé la question lors d'une projection-débat à Toulouse. Guy Chapouillié ${ }^{5}$ qui était présent nous l'a déconseillé et nous avons trouvé ses remarques extrêmement avisées. Il nous a convaincu de ne pas nous laisser aller à une vision esthétisante. Ces couleurs verdâtres ne dénaturaient pas le film, mais au contraire permettaient de rendre compte de la vie ouvrière dans ces lumières artificielles. Nous nous sommes rangés à son point de vue et d'une certaine manière, nous avons pris conscience que cela faisait partie d'une certaine conception de l'organisation du travail. Un film est un travail collectif : réalisateurs, opérateurs, monteurs, spectateurs contribuent à sa vitalité.

d'événements ou de situations ou de l'enregistrement de témoignages, revendiquées par certaines démarches ou disciplines : l'enregistrement, l'archivage ne suffisent pas à justifier le film sociologique. Penser l'image et son résultat signifiant en la composant : telles sont les ambitions du documentaire sociologique.

En même temps, l'image et le son, malgré toute l'attention apportée, ne peuvent pas tout dire. La sociologie du travail s'intéresse aussi à la subjectivité des acteurs et ici des ouvriers et des team leaders. D'où le recours à l'entretien filmé.

\section{Entretiens filmés et montage : faire sociologie}

Au-delà de l'impossibilité de comprendre les paroles enregistrées en atelier en raison $\mathrm{du}$ niveau sonore, nous souhaitions bénéficier de conditions favorables à la concentration des intéressés pour les conduire vers une attitude réflexive sur leur travail, sur les relations avec leurs pairs et avec les team leaders ou la hiérarchie, sur leurs aspirations, etc.

La salle de réunion qui nous a été dédiée le temps du tournage se présentait comme toutes les salles de réunion : froide, avec un éclairage zénithal au néon, des tables et des chaises impersonnelles. Heureusement, des dessins techniques sur les murs nous permettaient d'assurer la continuité avec l'atelier voisin que l'on entendait en bruit de fond. Le dispositif était donc assez contraint : les interviewés d'un côté de la table avec les dessins en fond d'image et nos deux caméras de l'autre côté. L'une des deux caméras tournait seule et l'opérateur (l'un de nous deux) choisissait l'angle et le cadrage ou modifiait les valeurs de plan selon les paroles de l'interviewé, tout en contrôlant le son. Le second posait les questions selon un script assez précis et relançait : nous avons fait évoluer le guide d'entretien afin d'aller toujours plus loin avec les monteurs impliqués quant à leur perception du travail et leur position dans la société américaine.

\subsection{Atteindre la parole}

31 Les entretiens préparés à partir de notre bonne connaissance des activités de montage automobile comportaient trois objectifs : partager la perception des ouvriers de leurs conditions de travail, connaître leur perception de l'organisation du travail (les principes de la lean production), débattre de la place qu'ils accordent à ce travail dans leur vie. 
32 En d'autres termes, ces entretiens filmés avaient pour objectif de sortir le plus rapidement possible du discours convenu (ce que les ouvriers interrogés pensaient que nous attendions d'eux) pour atteindre la parole, c'est-à-dire leurs convictions, l'intime, l'être-soi. Ce qui s'inscrit d'ailleurs dans un projet durable que nous avions amorcé avec une première recherche au début des années 1980 chez Renault avec le but de donner la parole à ceux qui restent silencieux, car leurs dires sont presque toujours médiatisés par l'écrit du chercheur qui place des verbatim dans sa démonstration. Si la gestuelle ouvrière a été amplement mise en images, la parole ouvrière l'est beaucoup moins. L'expression était assez libre et pratiquement jamais induite par quelque crainte de la hiérarchie ou de la direction de l'usine.

Ce média ne peut être affiché ici. Veuillez vous reporter à l'édition en ligne http:// journals.openedition.org/itti/630

Toute la conduite de l'entretien filmé invite l'interviewé à la réflexivité. Poser les bonnes questions c'est pousser l'interviewé à penser sa situation en se distanciant par rapport à celle-ci (au travail ou hors travail) : il s'agit de l'amener à mettre en tension son expérience et sa réflexion sur celle-ci, réflexion qui se construit bien souvent devant la caméra elle-même. Celui qui parle ne généralise pas, mais fait appel à ses projets personnels, à sa perception du monde et de son travail. L'entretien filmé se constitue ainsi comme un entre-deux: entre le singulier (qui réfère jusqu'à la psychanalyse) et le général (connu et convenu). Cet entre-deux qui parle de Soi est une libération de la parole et une reconnaissance ou une prise au sérieux de celle-ci car elle est offerte à des chercheurs qui consacrent du temps à la condition des ouvriers du montage : le respect de cette expérience du travail redonne une dignité à la parole. On peut parler de co-construction de la connaissance et du film. Y compris parce que la direction de l'usine accorde de l'importance et légitime l'interrogation en autorisant le tournage : ainsi la parole interrogée vaut quelque chose et chacun y met du sien pour enrichir l'échange qui traverse l'entretien filmé.

Dire le ressenti de son travail, donner sa perception du travail devant la caméra conduit à aller bien plus loin - ou autrement - que dans la discussion avec ses pairs ou avec ses proches. Plus précisément, c'est le pacte ou l'accommodement entre le sociologue et la caméra qui font monter et exprimer des pensées nouvelles à l'interviewé sur lui-même et sur sa condition dans sa situation particulière. Le couple sociologue-caméra pousse à la révélation d'un caché que chaque être humain porte en lui, mais qui demeure difficile à délivrer publiquement: ne pas réussir certains gestes dans les temps impartis, ne pas entrevoir de promotion possible dans l'entreprise, ne pas pouvoir reprendre d'études alors que les autres ouvriers le font pour échapper à leur condition. C'est peut-être ici la fonction maïeutique de ce couple sociologue-caméra qui fait de l'entretien filmé un outil de connaissance spécifique de valorisation de son être au monde (Merleau-Ponty, 1945, 505). Cette parole recueillie n'est pas un acte d'empathie $\mathrm{du}$ sociologue en direction de l'ouvrier qui se soumettrait à une injonction hiérarchique. L'explicitation du projet et sa présentation comme un désir de comprendre ce qui échappe à la perception immédiate du travail ouvrier ou de son rapport au travail, libèrent une autre parole, plus profonde, plus près du vécu, des tensions mais aussi des satisfactions qui sont au cœur des pratiques professionnelles. Rêves de chaîne expose les points de vue des monteurs, des syndicalistes, de la direction sur ce qu'est le travail ouvrier aujourd'hui ; mais au-delà, ce documentaire sociologique 
fait parler les imaginaires des ouvriers. Grâce en particulier à la magie du montage du film. Magie qui ne cherche pas à transformer ou à magnifier les dires, mais plutôt à faire émerger les implicites du travail, les résistances et les motivations.

\subsection{Du dérushage au montage}

36 Au-delà de la maîtrise technique des logiciels, le montage exige de bien en connaître les principes et d'en avoir une pratique qui évite les erreurs. D'où le recours à un professionnel : nous avons fait appel à un réalisateur et monteur de documentaires, Jean-Pierre Lenoir, avec qui nous échangions régulièrement sur nos expériences pédagogiques dans ce qui était alors le DESS Image et Société à l'Université d'Évry. En effet, le choix du monteur repose essentiellement sur la convergence de vues sur ce qu'est le documentaire et sur une confiance sans faille.

37 Le dérushage d'une quarantaine d'heures a d'abord conduit à un bout à bout de huit heures, dont une grande partie d'entretiens en anglais. S'il y a une spécificité du dérushage dans le documentaire sociologique, elle réside dans la rigueur des choix des plans et des séquences qui doivent traiter de l'objet questionné : pas de concessions pour des plans trop esthétisants ou trop éloignés de l'objet. Mais en même temps, l'attention portée aux images, aux sons et aux dires des interviewés conduit à des découvertes : des détails signifiants confirmant ou invalidant la thèse ou les thèses du film. Nous pensons ici aux déclarations frontalement anti-syndicales de plusieurs ouvrières que nous avons conservées, mais que nous avons associées à l'énoncé, par le négociateur de l'UAW, de ses récents succès en matière d'avantages sociaux. D'où la nécessité d'une transcription complète des entretiens afin de construire le déroulement progressif du film qui lui donnerait le maximum de contenus et de significations sociologiques. Transcription réalisée par Suzanne Newman, journaliste américaine exerçant à Paris: il fallait une oreille formée à l'accent et surtout aux idiomes américains pour décrypter ces entretiens. Car si les échanges étaient faciles en situation, il apparaissait que certaines nuances pouvaient échapper aux oreilles françaises. Nous avons alors réorganisé ces derniers autour de grands thèmes en questionnant et parfois en supprimant les redites d'un interviewé à l'autre, quelquefois en revenant aux images pour nous assurer de leur qualité.

Les chapitres et la structure du film commençaient à apparaître, mais nous étions bien loin du projet originel qui nous avait fait traverser l'Atlantique. Avec la même idée et les mêmes préoccupations sociologiques sous-tendant nos choix, le film prenait une autre tournure : telle est la réalité du documentaire sociologique. Entre le projet de départ, quelquefois scénarisé et le montage se glisse la réalité du matériau acquis pendant le tournage: des éléments souhaités qui sont absents, des masses de nouveautés et d'imprévus qu'il faut dompter pour qu'ils ne dénaturent pas le projet. En effet, il ne s'agit pas de se laisser aller à un opportunisme fondé sur le matériau existant, mais dans une voie pragmatique d'utiliser ce dont on dispose pour servir le projet. En même temps, il ne s'agit pas de rejeter ce qui ne correspond pas aux hypothèses de départ pour proposer un film lisse : bien au contraire, le film se doit de mettre en tension ces hypothèses avec un matériau qui peut les contredire. Ce qui est abandonné comme "résidu scientifique » dans nombre de recherches est conservé et réintroduit dans le documentaire sociologique. Tel est l'enjeu d'équilibrisme du 
montage : se référer au projet pour ne pas perdre le fil ni le sens du film et construire le documentaire à partir des matériaux, souvent contradictoires, dont on dispose.

Précédant la phase de montage proprement dite, nous avons recouru à des post-it assemblés en un montage virtuel, jusqu'à faire de leur utilisation une méthodologie systématique. Chaque personnage interrogé possédait sa couleur et chaque post-it résumait en une phrase une section de l'entretien (avec sa position dans le chutier6), selon une thématique correspondant au découpage du guide d'entretien filmé. Les postit de chaque auteur étaient ensuite collés sur différentes feuilles A2 matérialisant les chapitres du film, issus eux-mêmes du plan du guide d'entretien ; des post-it résumant les images/sons significatifs complétaient les post-it d'entretiens. Mais rapidement les chapitres ont exigé un rééquilibrage en déplaçant certains post-it d'une feuille à l'autre ; des post-it étaient découpés en sous-idées pour être bougés (d'où l'heureuse anticipation de placer verticalement la bande collante du verso des post-it). Dans chacun des chapitres, rapidement devenus multicolores, la progression des idées devait être organisée : à nouveau la fonction post-it favorisait les déplacements pour ordonner les idées. En même temps, des post-it restaient orphelins, révélateurs d'une marge à prendre en compte en les inscrivant dans tel chapitre à contre-courant des thèses partagées de celui-ci, ou en leur inventant quelques interstices signifiants. On voit ici combien l'écriture devient elle-même productrice de sens. Ainsi, la méthode des post-it et des couleurs projetait devant nous l'intégralité des matériaux dont on disposait: entretiens filmés et images de l'atelier. Elle révélait aussi la qualité des interventions de chaque personnage. S'agissait-il alors de les regrouper pour donner plus de profondeur à chaque propos, ou de les dissocier dans un jeu de questions-réponses entre eux. Cette phase fut d'ailleurs un moment d'intenses discussions sur le parti pris à retenir, chacune des logiques possédant son propre intérêt. Les deux pouvant d'ailleurs être présentes dans le montage final.

Cette opération de pré-montage à partir des post-it donne une vision globale du film, de ses composantes essentielles en termes de contenus et de distribution de la parole entre les différents intervenants. Le retour du monteur donne lieu à la confrontation avec les images pour leur qualité technique, mais surtout pour choisir celles qui possèdent le plus grand potentiel de significations para-langagières : il y a ici tout un travail sur les rapports paroles/images, sur la gestuelle de l'interlocuteur, sur les mimiques, sur les expressions du visage et sur le mouvement des mains. Filmer c'est donner à voir ce processus d'incorporation qu'a longuement étudié P. Bourdieu dans son analyse de l'habitus. Il ne s'agit pas ici d'un déterminisme social mais de la projection sur son corps de ce qui participe d'une intégration professionnelle en particulier lorsque celle-ci procède d'une trajectoire sociale, ancrée dans une tradition familiale dont le corps n'a pu se dessaisir. Cela avait d'ailleurs contribué au choix paradoxal de notre premier projet et explique probablement que nous ayons été conquis par les deux premières femmes que nous envisagions de retenir comme personnages. 


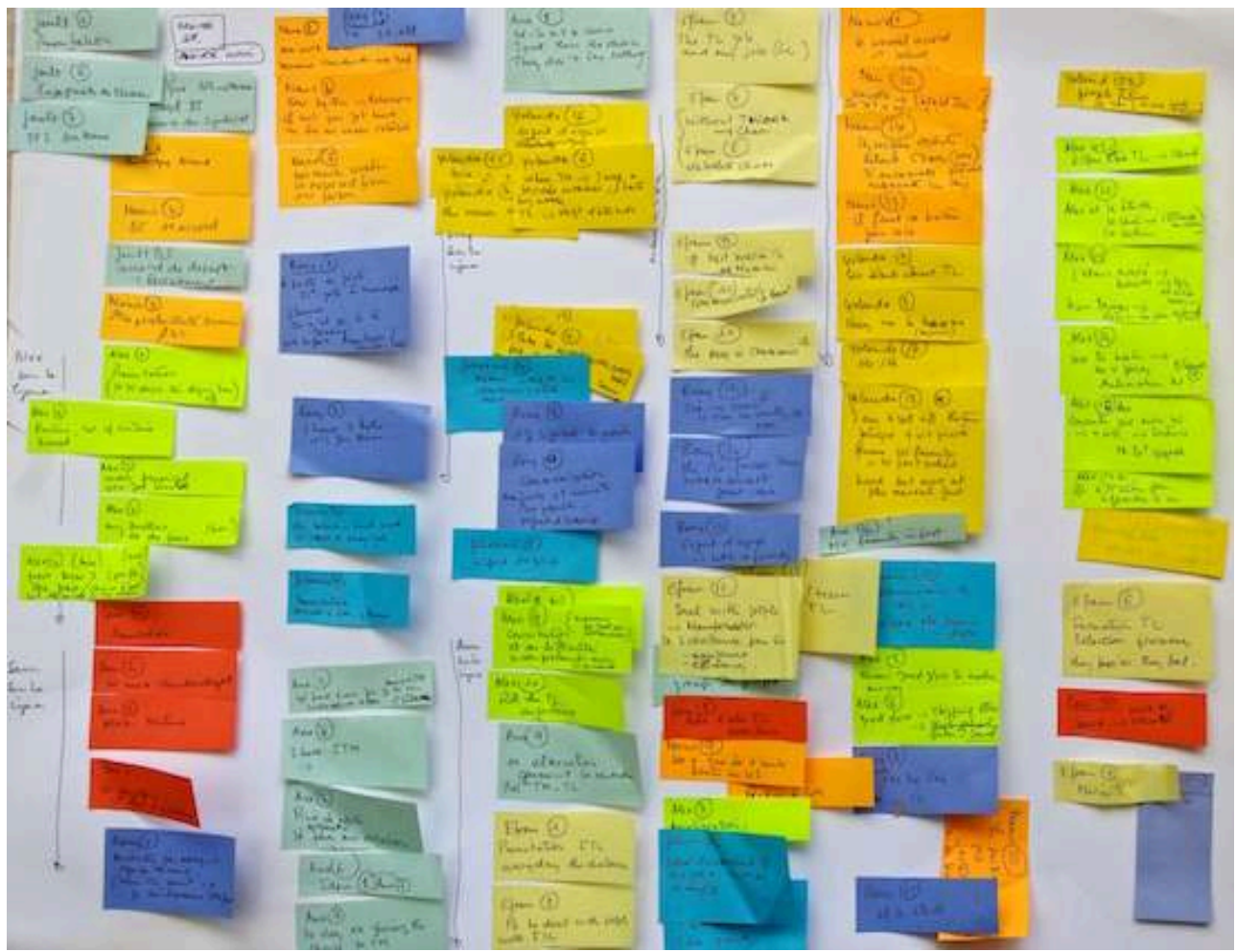

Déjà présente dans les post-it, éclate ici la récurrence de la dureté du travail, des émotions, mais aussi des aspirations et des rêves. À force de revoir les images, chacun y découvre de nouvelles potentialités. Nous faisons corps avec la matière qui est devant nous. Nous élaguons les redites, nous choisissons les séquences montrant celui ou celle qui a la parole la plus pertinente, à la fois sur le fond et dans la forme. Peu à peu émerge le fil rouge (et bientôt le titre du film, Rêves de chaîne) qui résume toute la situation et la condition ouvrière : même si les salaires sont corrects ( 20 \$ de l'heure), les conditions de travail poussent tous ceux qui le peuvent à imaginer une sortie de la ligne de montage. Ce qui corrobore les déclarations de la militante que nous avons rencontrée mais qui a refusé d'être filmée : l'atelier Tacoma, c'est l'enfer; pour les ouvriers des anciens ateliers, les cadences de travail du nouvel atelier sont bien plus dures que celles qu'ils connaissent.

Toutes les conditions sont réunies pour réaliser le montage qui durera deux mois intensifs. L'ordonnancement "théorique » via la méthode des post-it est bouleversé parce qu'il faut à la fois ne pas monter des plans d'entretiens trop courts que le spectateur ne pourra pas suivre ou comprendre et parce qu'il faut tenir compte a minima des contraintes picturales et sonores dans l'enchaînement des plans. Pourtant, les post-it nous ont permis de posséder, mémoriser et connaître parfaitement les contenus des images et des paroles afin que nous sachions le film que nous voulions et que nous pouvions monter avec les matériaux disponibles. Cette mémoire collective des rushes a été essentielle à la concrétisation du montage.

Une attention particulière est accordée au générique de début qui donne le ton et qui pose la problématique du film en le contextualisant sans ne rien dévoiler de celui-ci. C'est la raison pour laquelle le film commence par une longue introduction sur le statut de la voiture aux États-Unis et en Californie plus particulièrement: autoroutes 
surchargés et embouteillages, ponts autoroutiers de San Francisco et défilement des gratte-ciels qui font des villes nord-américaines des villes excluant le piéton au bénéfice de l'automobile. Mais celles-ci meurent aussi: nous avions filmé sur une autoroute un camion rempli de carcasses écrasées. Ce qui préparait bien le plan sur le blason Toyota, comme allégorie de l'affaiblissement de l'industrie automobile américaine face aux firmes japonaise. Tout se passe en Californie, État dans l'État, toujours en expansion et en travaux: les bulldozers en témoignent avec leur rugissement, au milieu d'un arrosage systématique des terrains à travailler alors que l'eau manque! Un panoramique extérieur de l'usine et nous pouvons entrer dans l'usine avec une séquence des ouvriers au travail, immédiatement suivie de la parole d'un syndicaliste qui plante le décor de la réouverture de l'usine avec les nouvelles contraintes imposées par Toyota aux monteurs qui ont été réembauchés. Le montage est l'occasion d'une réflexion sur la dimension synthétique et elliptique du documentaire sociologique.

Ce média ne peut être affiché ici. Veuillez vous reporter à l'édition en ligne http:// journals.openedition.org/itti/630

Tout au long du montage, nous avons apporté un soin particulier à la bande-son : qualité d'une part et significations manifestes ou latentes d'autre part. Le niveau sonore a été rendu tout au long des séquences en atelier, avec ses klaxons quasi permanents, plus aigus, d'appel à l'aide. Il s'agit de signifiants très forts, mais ils ne sont compris que par les spectateurs qui connaissent les ateliers de montage automobile ${ }^{7}$. Toujours à propos du générique, c'est le seul endroit ou apparaît la musique: pour renforcer l'entrée en matière, nous avons conservé la musique de l'autoradio qui accompagnait le tournage des panoramiques des autoroutes dans la baie de San Francisco : rythmée et répétitive, voire obsédante, elle préfigure le travail de l'atelier.

46 Au regard du matériau collecté le documentaire suit le fil rouge des aspirations et des rêves des ouvriers et des team leaders en se structurant autour de la parole de ceux-ci, avec des images et des sons de l'atelier qui ne sont pas là pour illustrer un propos, mais qui fonctionnent de façon autonome. Elles sont pour nous une autre manière de dire le travail ouvrier : elles plongent une partie de leurs significations dans les entretiens, lesquels peuvent aider le spectateur à mieux lire les images/sons et à les interpréter avec une meilleure connaissance du terrain qu'il n'avait auparavant. Le film achevé, il nous fallait le restituer aux participants et à la direction de l'usine d'accueil : sans que nous fassions à nouveau le voyage, nous l'avons fait circuler parmi les ouvriers et les personnages filmés qui l'ont accepté en l'état, alors que nous redoutions que certaines déclarations assez sévères qu'ils font à l'encontre de la direction sur la dureté du travail leur paraissent mal venues dans le film. Il n'en a rien été. De son côté, la direction de la communication a trouvé le film «riche et équilibré». La diffusion en était devenue possible. 


\section{Retour sur une démarche : de la spécificité du film sociologique}

47 À partir de la préoccupation permanente, pour ne pas dire obsessionnelle de fabriquer des images ou de donner aux mouvements (Maillot, 2000) des significations sociologiques, le documentaire sociologique inclut toujours plus d'informations qu'il n'y paraît au premier abord. Et l'on en redécouvre à chaque visionnage. Il est dommage que l'on ne regarde pas plusieurs fois le documentaire comme on relit un livre ou bien que l'on retourne en arrière pour établir une relation avec ce que l'on est en train de lire. Le statut de l'image, pourtant omniprésente dans nos sociétés est celui d'une chose évanescente, contrairement au texte qui est perçu comme plus solide, plus dur.

\subsection{Puissance des images/sons}

Pourtant, tout ce que comportent les gestes, les mimiques, les positions corporelles, les intonations de la voix, les hésitations de la parole ou les silences, au travail comme dans l'entretien, sont indicibles pour les acteurs eux-mêmes comme pour les experts. Par exemple, la fraction de seconde d'arrêt des poseurs de lunette-arrière au-dessus de la carrosserie est bien «parlante » de ce que nous pouvons en dire ici : ses signifiants vont jusqu'à interroger la formation: comment acquiert-on cette dextérité ? Y-a-t-il un meneur -comme dans la danse- parmi les deux ouvriers? Et quand l'un des deux ouvriers va occuper un autre poste, comment l'adaptation du nouvel arrivé a lieu?

Le documentaire comprend aussi de nombreux non-dits, tout juste esquissés qui peuvent venir du montage lui-même. Par exemple ici, on peut trouver une belle hiérarchie des rêves. Le chef de groupe afro-américain semble avoir trouvé sa voie: après avoir été militaire et avoir participé à des opérations guerrières, il découvre un milieu où l'organisation, tout aussi rigide que l'armée sur le fond, doit être mise en œuvre par la conviction plutôt que par l'ordre. À l'opposé, l'ouvrier philippin, récemment arrivé sur le continent, ne peut espérer aucune évolution de sa condition : il le dit sur un ton qui ne laisse pas indifférent. Les team leaders - toutes femmes, ce qui est aussi un sujet d'interrogation - sont sorties de la ligne de montage, du flux tendu, pour l'essentiel de leurs activités : la plupart d'entre elles n'aspirent pas à changer de fonction. Seule l'une d'entre elles - espérant percer comme chanteuse en allant à New York - rejoint le groupe des ouvriers qui expriment clairement le fait qu'ils sont là, de passage. Ils poursuivent des études et aspirent à quitter l'usine pour des horizons plus cléments. C'est aussi une invitation à analyser la politique managériale de gestion de la main d'œuvre : au-delà d'un noyau dur d'ouvriers, recruter des jeunes qui partiront bientôt supprime le poids des retraites et allège l'entreprise du handicap du vieillissement des ouvriers-monteurs, moins productifs au-delà de la quarantaine...

Ce média ne peut être affiché ici. Veuillez vous reporter à l'édition en ligne http:// journals.openedition.org/itti/630

51 La sociologie filmique n'a pas pour objectif principal de présenter une enquête ou ses résultats sonores ou imagés. Pour nous, l'enquête a lieu très en amont et se fait selon les méthodes traditionnelles de la sociologie (ou des autres disciplines associées aux méthodes sociologiques). Elle est nécessairement approfondie afin que le réalisateur 
connaisse suffisamment bien son terrain pour ne pas être pris au dépourvu par les incidents de tournage, tout en utilisant les bonnes surprises offertes par tout travail de terrain. Bien sûr, cette enquête approfondie peut - et l'on pourrait dire devrait - être accompagnée de prises de vue (fixes ou animées), voire de prises de sons. Lesquelles ne seront qu'exceptionnellement reprises dans le montage final mais permettent de mieux préparer le tournage : à partir de celles-ci le réalisateur choisit ses angles de vue, c'està-dire aussi ses points de vue ${ }^{8}$. En effet, chaque angle de vue exprime une relation du sociologue-cinéaste à son objet: cadrage à hauteur d'œil, en plongée, en contreplongée, plan rapproché, plan large font habituellement référence à des rapports de proximité, d'empathie, de domination, de soumission, etc. De même, les mouvements de caméra, le choix des lumières traduiront les perceptions que le cinéaste souhaite partager à condition qu'il possède une très bonne connaissance du terrain. Enfin, le travail d'enquête préalable permet d'inventer le dispositif filmique indispensable à toute narration cinématographique ${ }^{9}$.

La sociologie filmique se propose de combiner trois objectifs étroitement imbriqués c'est d'ailleurs cette imbrication qui fait sens - que l'on ne distingue ici que pour l'exposé :

- Recueillir et enregistrer des données relatives à un objet sociologique circonscrit (images et sons), sans oublier de prendre en compte autant que faire se peut le contexte du travail de terrain, la conceptualisation et les relations de causalité existant entre les faits sociaux (au sens large, c'est-à-dire n'excluant ni les facteurs économiques, ni les dimensions subjectives des situations). La difficulté ultérieure réside ici dans la nécessité de trouver un procédé narratif afin de donner une attractivité au film. Celle-ci confère son sens au projet dont l'objectif premier est de rendre visible une partie de la dimension sociale du travail qui reste encore aujourd'hui un monde clos.

- Diffuser autrement qu'à travers le support papier (ou l'écrit sur l'Internet) les connaissances sociologiques: ce qui pourrait signifier un public plus large, mais ici la question des diffuseurs reste posée, entre les formats de plus en plus contraints des chaînes de télévision et les incertitudes quant aux publics de l'Internet.

- Produire des connaissances sociologiques tout au long du processus de réalisation du film. En particulier, l'image et le son permettent de saisir et de communiquer les dimensions du sensible propres aux situations sociales. Comme nous l'avons vu plus haut, au-delà de la seule question du sensible, les choix dans le tournage (position de la caméra et des micros, nature du plan, déplacements de la caméra, éclairages, etc.), puis le long travail sur l'image et le son que requièrent le dérushage et le montage conduisent le réalisateur à s'interroger en permanence sur le saisissement de sens par les images, par la mise en rapport des sons et des images, par la mise en rapport des plans et des séquences, etc.

\subsection{Cinéma et sociologie}

53 C'est essentiellement autour de ces questionnements que se constitue peu à peu une épistémologie de la sociologie filmique qui doit tenir compte à la fois :

- De l'histoire du cinéma et de l'interrogation permanente sur le processus par lequel le sens vient aux images et aux sons: comment «on pense en cinéma» (Deleuze, 1983), en particulier lorsque le mouvement s'associe à l'image et au plan (Maillot, 2000, 52 ; Durand et Sebag, 2015). 
- Des préoccupations sociologiques fondamentales réorganisées au prisme de l'image et du son (Sebag 2011, 2012) : poser un questionnement, argumenter des réponses et conduire le spectateur vers des conclusions. d'une histoire et de préoccupations largement hétérogènes, avec des métiers qui ne se sont guère croisés car ils recourent à des outillages différents. L'objectif est de construire une convergence. Cela passe par une réflexion sur les multiples approches de la sociologie et sur les modes de questionnements qui permettent une rencontre entre les deux écritures. C'est le fruit d'un long travail de compréhension respective des complémentarités entre ce qui apparaît comme deux visions du monde : l'art et la science. Y-a-t-il compatibilité ? Au premier abord la réponse était plutôt négative de part et d'autre. On peut en donner les lignes générales suivantes :

- Pour les cinéastes, la compréhension du monde et de la société allait de soi, il suffisait de lire, d'écouter, d'être au monde pour s'en saisir et construire des récits aux fondements réels ou imaginaires. Le langage de la sociologie était aride et incompatible avec celui du cinéma, puisque le leit motiv de ce dernier était sensibilité, émotion.

- Pour les sociologues, malgré des décennies de tentatives de rapprochement ${ }^{10}$ le scepticisme était aussi grand. Introduire de l'émotion, de la sensibilité dans une approche scientifique c'était déconstruire plus d'un siècle d'autonomisation de la discipline et d'élaboration conceptuelle.

Notre travail a consisté non pas à convaincre les un.e.s et les autres de la possibilité d'une démarche commune, mais de construire celle-ci en nous appuyant sur des recherches issues de plusieurs disciplines. Le cheminement vers la sociologie filmique ne pouvait se faire sans l'accompagnement théorique de nombre d'auteurs. La dimension opératoire de leurs thèses nous est apparue en ce qu'elle rompait souvent avec une vision trop positiviste de la sociologie. Elle rendait possible la mise en présence sinon la mise en scène des différents protagonistes d'une recherche. Cette forme de recherche et d'écriture par l'image et le son nous a conduits à poser la question de notre subjectivité dans le rapport à notre objet tout en construisant notre distance à celui-ci. Les relations sociales n'étaient plus une abstraction dont on allait rendre compte ou que l'on allait décrire mais elles devenaient des faits observables par le spectateur qui pouvait aussi s'interroger sur notre propre regard. Il en est de même de l'espace environnant qui situe la parole des sujets et aide à leur compréhension. Ainsi, la démarche entreprise résulte d'un processus cumulatif. C'est une mise en relation des connaissances issues de différentes disciplines et de différents champs qui à un moment donné paraissent efficaces.

De plus, dans la démarche de la sociologie filmique, ce qui importe est de voir toutes les possibilités qu'offrent ces recherches associées à une compréhension et à une certaine maitrise de l'écriture cinématographique pour proposer une approche à la fois sensible, conceptuelle et incorporée de l'écriture sociologique. Lier la forme et le fond, suppose une réflexion ad hoc, ou ce qui a été nommé plus haut «le dispositif » c'est-à-dire un travail d'adaptation de la narration au questionnement et aux personnages retenus ${ }^{11}$.

C'est en effet à partir du langage cinématographique que les sociologues réaliseront des documentaires scientifiques de qualité à diffuser vers de nouveaux publics. La forme, rappelons-le, exige cette maîtrise des outils du cinéma (et aujourd'hui de la vidéo) pour que le documentaire sociologique conduise le spectateur à s'interroger et à penser. Car l'une des volontés de la sociologie filmique est d'écarter les films à thèse 
simplificateurs, autre avatar du prêt à penser. En effet, tout en refusant la neutralité axiologique en sociologie, le documentaire sociologique conduit le spectateur à penser, c'est-à-dire à construire sa propre réflexion pour répondre à la ou aux questions posées par le sociologue-cinéaste ; lequel propose des éléments de réponse par les images/sons ou à travers des entretiens qui relatent son point de vue sans en faire un absolu. Cet équilibre ou plutôt cette tension constitue les raisons d'être du documentaire sociologique qui fait du spectateur, un acteur.

Dans cet article nous avons tenté de montrer les potentialités qu'offre la sociologie filmique. Quelle compréhension et quelle sensibilité autres rendait-elle possible? Ce que nous avons découvert, pouvait-il être perçu par le spectateur ou suggéré par un autre support? Au cours de ce long processus qu'est la réalisation d'un film fondé sur des questionnements sociologiques, nous sommes toujours restés réceptifs aux découvertes que favorise l'usage de la caméra. Et celles-ci furent nombreuses :

- L'une des premières vertus est de mettre en évidence la très bonne analyse de leur situation, de leurs conditions de travail par les ouvriers-monteurs sans que celles-ci soient médiatisées par le résumé, le verbatim ou la conceptualisation d'un journaliste, d'un syndicaliste ou d'un chercheur. C'est cette réflexivité qui nous semble avoir été mise en valeur lors des entretiens filmés.

- La mise en évidence de leur parfaite élocution, loin de l'ouvrier muet du fordisme tel qu'il fut présenté pendant un siècle.

- L'aisance du corps jeune dans ce type de travail qui constitue un élément du langage cinématographique tout en montrant la capacité de la direction à susciter d'autres aspirations chez l'ouvrier afin d'organiser un turnover systématique pour ne plus avoir à faire face aux problèmes inhérents au vieillissement de la main d'œuvre.

Ainsi, la sociologie filmique ouvre d'innombrables possibles, à condition qu'elle cultive un public intéressé par l'image et le son, prêt à une lecture affûtée de ceux-ci : «le spectateur émancipé » dont parlait Rancière (2008). Mais ceci ne tient qu'à la condition que les sociologues se préoccupent sérieusement d'acquérir la maitrise du langage cinématographique sans abandonner le fond de leur métier ; c'est-à-dire qu'ils soignent la construction du sens dans les images et les sons. Sans oublier la préoccupation esthétique qui fera aimer leurs réalisations.

\section{BIBLIOGRAPHIE}

Adler P. (1998), « Nummi : de l'autonomie du travail à la socialisation de la production ?», in J.-P. Durand, P. Stewart et J.J. Castillo, L'avenir du travail à la chaîne. Une comparaison internationale dans l'industrie automobile, Paris, La Découverte.

Bourdieu P. (1986), «L'illusion biographique », Actes de la recherche en Sciences Sociales, Numéro thématique, L'illusion biographique, Vvol. $62, \mathrm{n}^{\circ} 1$.

Debray R. (1992), Vie et mort de l'image, une histoire du regard en Occident, Paris, Gallimard.

Deleuze G. (1983), Cinéma, Tome 1, L'image-mouvement, Paris, Éditions de Minuit. 
Deleuze G. (1985), Cinéma Tome 2, L'image-temps, Paris, Editions de Minuit.

Durand J.-P. et Nicolas Hatzfeld (2002), La chaîne et le réseau. Peugeot-Sochaux, ambiances d'intérieur, Lausanne, Editions Page 2.

Durand J.-P. (2004), La chaîne invisible. Travailler aujourd'hui : du flux tendu à la servitude volontaire, Paris, Le Seuil.

Durand J.-P. et J. Sebag (2015), « La sociologie filmique : écrire la sociologie par le cinéma? », L'Année sociologique, vol. 65, $\mathrm{n}^{\circ} 1$.

Elias N. (1993), Engagement et distanciation. Contributions à la sociologie de la connaissance, Paris, Fayard.

Garfinkel H, (1967), Studies in Ethnomethodology/Recherches en ethnométhodologie, (traduction française - 2007), Paris, PUF.

Goffman E. (1973), La mise en scène de la vie quotidienne 1, La présentation de soi, Paris, Les Éditions de Minuit.

Goffman E. (1974), La mise en scène de la vie quotidienne 2, Les rites d'interaction, Paris, Éditions de Minuit.

Kracauer S. (1973), De Caligari à Hitler, une histoire psychologique du cinéma allemand, Lausanne, L’âge d'homme.

Lefebvre H. (1974), « La production de l'espace », l'Homme et la société, vol. 31, p. 15-32, Paris. [En ligne] URL : http://www.persee.fr/doc/homso_0018-4306_1974_num_31_1_1855

Leveratto J.-M. (2009), « La Revue Internationale de Filmologie et la genèse de la sociologie du cinéma en France », Cinémas, vol. 19, n 2-3. [En ligne] URL : https://www.erudit.org/fr/revues/ cine/2009-v19-n2-3-cine3099/037553ar/

Lorino P. (1987), Les Saturniens. Quand les patrons réinventent la société, Paris, La Découverte.

Maillot P. (2000), «Qu'est-ce que penser en cinéma », CinémAction, n 94, 48-55.

Maillot P. (2012), « L'écriture cinématographique de la sociologie filmique. Comment penser en sociologue avec une caméra? », La Nouvelle Revue du Travail, n 1. [En ligne] URL : http:// nrt.revues.org/363

March J.G. et Simon H.A., ([1958],1966), Les organisations. Problèmes psychosociologiques, Paris, Dunod.

Merleau-Ponty M., (1945), Phénoménologie de la perception, Paris, Gallimard.

Morin E. (1956), Le cinéma ou l'homme imaginaire, Paris, Éditions de Minuit .

Naville P. (1966), «Instrumentation audio-visuelle et recherche en sociologie », Revue Française de Sociologue, VII, 158-168, Paris. [En ligne] URL : http://www.persee.fr/doc/

rfsoc_0035-2969_1966_num_7_2_1113

Rancière J. (2000), Le partage du sensible : Esthétique et politique, Paris, La Fabrique.

Rancière J. (2008), Le spectateur émancipé, Paris, La Fabrique.

Ricœur P ([1983], 1991), Temps et récit 1 : L’intrigue et le récit historique, Paris, Seuil (Coll. Points Essais)

Ricœur P ([1984], 1991), Temps et récit 2. La configuration du temps dans le récit de fiction, Paris, Seuil (Coll. Points Essais) 
Ricœur P. ([1985], 1991), Temps et récit : Le temps raconté, Paris, Seuil (Coll. Points Essais)

Sebag J. (2010), « Regards sur la sociologie visuelle à partir d'une lecture de l'ouvrage "La théorie du film, la rédemption de la réalité matérielle de Siegfried Kracauer" ", in Journées d'études organisées par Monique Peyrière, Siegfried Kracauer, Actualité cinématographique, EHESS, 22-23 juin 2010.

Sebag J. (2011), « De la sociologie visuelle à la Sociologie filmique », in Gehin JP. et H. Stevens, (dir.), Images du travail, travail des images, Rennes, Presses Universitaires de Rennes.

Sebag J. (2012), « Sociologie filmique et travail », La Nouvelle Revue du Travail, $\mathrm{n}^{\circ}$ 1. [En ligne] URL: http://nrt.revues.org/383

Womack J. P., D. T. Jones et D. Roos (1992), Le système qui va changer le monde, Paris, Dunod, [1990].

\section{NOTES}

1. Groupe d'Études et de Recherches Permanent sur l'Industrie et les Salariés de l'Automobile.

2. Dans les usines syndicalisées de l'industrie automobile nord-américaine, l'employeur est tenu de reprendre les salariés licenciés à la suite de difficultés économiques de l'entreprise, par ordre d'ancienneté.

3. De façon contradictoire, l'atelier possédait un certain nombre d'équipements efficaces d'aide à la manutention qui allégeaient l'effort des ouvriers, mais les portes des véhicules n'étaient pas démontées, comme dans d'autres usines. Ce qui accroissait les difficultés d'accès à l'intérieur des cabines pour visser ou pour agrafer.

4. De fait il n'y a guère que dans les usines japonaises que l'on peut saisir par le film la forte intensité du travail, car celle-ci apparaît nettement quand on la compare au travail dans d'autres pays. Voir par exemple les plans filmés en usine dans notre documentaire sociologique Nissan, une histoire de management. Manuela Frésil a pu en saisir un aspect dans la séquence de l'emballage des poulets dans son film Entrée du personnel $(2011,59$ ').

5. Fondateur de l'École supérieure d'audiovisuel (ESAV) de Toulouse.

6. Le chutier est une sorte de base de données du logiciel de montage dans laquelle le monteur range et ordonne les séquences et les plans (images et sons) avec des repères précis pour les retrouver afin de les assembler dans le montage final.

7. Il en est quasiment de même des panneaux aériens qui, à travers deux nombres, indiquent le retard, très rarement l'avance, sur les prévisions.

8. Voir, pour cette question du point de vue, le colloque international organisé par le Centre Pierre Naville et l'AFS sur «Le point de vue dans la vie quotidienne », 28-30 septembre 2016 (http://gt47.hypotheses.org).

9. Voir sur l'importance de la construction du dispositif dans le documentaire, Maillot, 2012.

10. Dans un article paru dans la revue Cinéma, Jean-Marc Leveratto (2009) revient sur les projets qui ont sous-tendu la Revue Internationale de Filmologie (RIF) qui parait dès la fin de la Seconde Guerre Mondiale à laquelle contribueront Friedmann et Morin. Cette revue marque la rencontre entre la sociologie et le cinéma. Le rapprochement visait essentiellement à rendre compte de la capacité de la sociologie à faire du cinéma un fait sociologique et non à initier directement une sociologie par le cinéma. Un auteur majeur de cette démarche, Siegfried Kracauer aux liens ténus avec l'École de Francfort, y participa de même que Morin dont on connaît les écrits sur le cinéma et le rôle qu'il joua dans une sociologie par le cinéma avec Chronique d'un été (1961).

11. Le fond est entendu comme l'appropriation des résultats de recherches sur le thème concerné et la capacité à produire de nouvelles connaissances dans un langage cinématographique. 


\section{RÉSUMÉS}

Les auteurs interrogent leur pratique de sociologues-réalisateurs à propos de Rêves de chaîne, documentaire sociologique réalisé au début des années 2000 dans une usine de montage automobile en Californie, joint venture de Toyota et General Motors.

Dans cet article réflexif, les auteurs examinent chacune des grandes phases de réalisation du film, de l'enquête préalable au tournage jusqu'au montage. Quelle est la spécificité de la démarche sociologique dans la construction du film? Quels emprunts nécessaires au langage cinématographique? Quels sont les rapports avec les professionnels du cinéma ? Comment vient le «sens sociologique » aux images/sons et quelle place accorder au montage?

L'article fait état des difficultés rencontrées, des solutions adoptées au cours de ce long processus de réalisation d'un documentaire et des apports de la démarche cinématographique dans la production scientifique en sociologie. L'article se situe dans la perspective du développement de la sociologie filmique dont il interroge la capacité à faire sens, à rendre visibles les paroles, les pratiques, les corps, les gestes des personnages qui sont au fondement d'une recherche.

The paper scrutinises the actions that the authors took as sociologist film-makers responsible for producing "Dreams on line" (Rêves de chaîne), a sociological documentary made in the early 2000s in a California automotive plant run by a Toyota and General Motors joint-venture.

It is a reflective article examining each of the film's main production phases, from pre-filming preparations through the final edits. Questions pertain to the specificity of a sociological approach to film-making; the borrowing of cinematographic language ; relations with cinematic professionals; the imbuing of images and sounds with sociological meaning; and the significance of the final edit.

The article looks at the challenges that film-makers face, as well as the solutions they find throughout the long process of making a documentary. It also asks to what extent the cinematographic approach can enhance scientific sociological production. The ultimate purpose is to further develop the sociology of film by scrutinising the discipline's ability to create meaning and add to the visibility of the words, practices, bodies and gestures of the individuals being subjected to investigation

\section{INDEX}

Mots-clés : sociologie filmique, industrie automobile, Toyotisme, travail ouvrier, réflexivité sociologique, point de vue, entretien filmé

Keywords : filmic sociology, cinematic sociology, automotive industry, Toyotaism, shopfloor work, sociological reflexivity, point of view, filmed interview

\section{AUTEURS}

\section{JEAN-PIERRE DURAND}

Jean-Pierre Durand est Professeur émérite de Sociologie à l'Université d'Évry Paris-Saclay où il a fondé le Centre Pierre Naville (cpn.univ-evry.fr). Il est l'auteur de plusieurs ouvrages de sociologie générale (dont Sociologie contemporaine, Vigot, 2006) et La Sociologie de Marx, La Découverte, 2018). Il a fondé en 2012 et dirige la Nouvelle Revue du Travail devenue en quelques années une des revues de référence (https://journals.openedition.org/nrt/). En sociologie du 
travail il a notamment publié : La chaîne invisible. Du flux tendu à la servitude volontaire (Le Seuil, 2004) et La fabrique de l'homme nouveau. Travailler, consommer et se taire ? (Le Bord de l'Eau, 2017). Il a réalisé plusieurs documentaires sociologiques avec Joyce Sebag.

\section{JOYCE SEBAG}

Joyce Sebag est Professeur émérite de Sociologie (Centre Pierre Naville, Université d'Evry). Elle a publié de nombreux articles sur l'écriture cinématographique en sociologie dont « Genèse d'une écriture documentaire : renouer avec la connaissance dialoguée », Revue Entrelacs, Toulouse, 2016. « La sociologie filmique : écrire la sociologie par le cinéma? », L’Année sociologique, 2015/1, « Sociologie filmique et travail », La Nouvelle Revue du Travail, 1/2012 (https://journals.openedition.org/nrt/383), « Le temps de travail au cinéma : comment représenter l'intangible ? » in Claude Durand, Temps et temps de travail, Paris, De Boeck, 2001. Elle est co-responsable du groupe de recherche Sociologie visuelle ou filmique au sein de l'Association française de Sociologie et dans l'European Sociological Association. Elle a réalisé plusieurs documentaires sociologiques avec J.-P. Durand : Rêves de chaîne (26'), Nissan, une histoire de management (34'), Boston, 50 ans d'Affirmative Action (52') et Femmes en banlieue, (réalisé avec Sylvia Calle, Pierre Linhart, montage Christophe Nowak), 30'. 